Benchmarks

\section{$A$ rapid and cost-effective method for sequencing pooled cDNA clones by using a combination of transposon insertion and Gateway technology}

Takeya Morozumi ${ }^{1,2}$, Daisuke Toki ${ }^{1,2}$, Tomoko Eguchi-Ogawa ${ }^{1,3}$, and Hirohide Uenishi ${ }^{1,3,4}$

${ }_{1}^{1}$ Animal Genome Research Program, Tsukuba, Japan, ${ }^{2}$ Animal Research Division, Institute of Society for Techno-innovation of Agriculture, Forestry and Fisheries, Tsukuba, Japan, ${ }^{3}$ Agrogenomics Research Center, National Institute of Agrobiological Sciences, Tsukuba, Japan, and ${ }^{4}$ Division of Animal Sciences, National Institute of Agrobiological Sciences, Tsukuba, Japan

\section{BioTechniques 51:195-197 (September 2011) doi 10.2144/000113737}

Keywords: cDNA sequencing; transposon shotgun sequencing; clone pooling; automation

Supplementary Material for this article is available at www.BioTechniques.com/article/113737.

Large-scale cDNA-sequencing projects require an efficient strategy for mass sequencing. Here we describe a method for sequencing pooled cDNA clones using a combination of transposon insertion and Gateway technology. Our method reduces the number of shotgun clones that are unsuitable for reconstruction of cDNA sequences, and has the advantage of reducing the total costs of the sequencing project.

Several strategies for large-scale cDNA sequencing have been developed, such as primer-walking (1) and transposon shotgun (TPS) sequencing (2). In the primer-walking method, cDNA clones carrying GC-rich or repetitive sequences often cannot be completely sequenced. Furthermore, primer-walking requires procedures for primer design and rearraying of DNA samples that are not suitable for automation. The TPS sequencing strategy can easily be

automated, because it does not require custom sequencing primers; however, it requires relatively expensive reagents and generates many "invalid" clones that carry transposons inserted in the vector sequence. Previous studies have demonstrated that the cost of sequencing projects can be reduced by pooling plasmids before transposon introduction (3) and that shotgun clones with transposons inserted in the vector sequence can be eliminated by Gateway technology (Invit-

Table 1. Transposon insertion rates in the vector or insert sequences of cDNA-carrying plasmids.

\begin{tabular}{lcc|} 
& BP reaction \\
& No (with 192 TPS clones) & Yes (with 96 TPS clones) \\
\hline Within insert & $1267(35 \%)$ & $1754(93 \%)$ \\
\hline Within vector & $2380(65 \%)$ & $136)$ \\
\hline $\begin{array}{l}\text { Transposon shotgun (TPS) clones were generated from } 20 \text { cDNA pools, each of which consisted of } 12 \\
\text { cDNA clones. Each pool was processed or not processed by the BP reaction, generating } 96 \text { and } 192 \text { TPS } \\
\text { clones, respectively. If any bases in either of the sequencing reads were not of high quality (Phred quality } \\
\text { value }<14), \text { the TPS clone was omitted from the analysis. }\end{array}$ \\
\hline
\end{tabular}

rogen, Carlsbad, CA, USA) $(4,5)$. Here we demonstrate the efficacy of a rapid and cost-effective method for cDNA sequencing utilizing a combination of plasmid DNA pooling and translocation of transposon-inserted cDNA sequences by Gateway technology.

Pig cDNAs that had been constructed by the oligo-capped method and subjected to expressed sequence tag (EST) analysis $(6,7)$ were cloned into the Gateway-compatible pCMVFL3 vector (Invitrogen) $(6,8)$. Plasmid DNA was prepared as described previously $(3,9)$. Twelve plasmid DNAs (approximately $1 \mu \mathrm{g}$ each) were pooled and subjected to transposon insertion using an EZ-Tn5 <KAN2> insertion kit (Epicentre, Madison, WI, USA) in a total volume of $5 \mu \mathrm{L}$ instead of the manufacturer's suggested $10 \mu \mathrm{L}$. The resultant mixture was adjusted to $500 \mu \mathrm{L}$ with TE buffer (1 mM EDTA, pH 8.0), desalted by an Amicon-Ultra-0.5 mL $50 \mathrm{~K}$ column (Millipore, Billerica, MA, USA) and concentrated to $30 \mu \mathrm{L}$. The insert transfer reaction was conducted at $25^{\circ} \mathrm{C}$ overnight with $3.5 \mu \mathrm{L}$ mixture recovered from the column, $75 \mathrm{ng} / \mu \mathrm{L}$ pDONR-Zeo (Invitrogen) linearized with $E c o$ RI, and $1 \mu \mathrm{L}$ BP clonase II enzyme mix (Invitrogen). The resultant mixture was processed with proteinase $\mathrm{K}\left(1 \mu \mathrm{g}\right.$; Invitrogen) at $37^{\circ} \mathrm{C}$ for $10 \mathrm{~min}$ and then deposited on a Millipore $\mathrm{V}$ series membrane (25-nm pore) floating on sterilized water to dialyze for $1 \mathrm{~h}$ at room temperature.

Using a Gene Pulser II Electroporator (Bio-Rad Laboratories, Hercules, CA, USA), $10 \mu$ L ElectroMAX DH10B cells (Invitrogen) were transformed with $3 \mu \mathrm{L}$ dialysate at $1.8 \mathrm{kV}, 200 \Omega$, and $25 \mu \mathrm{F}$ in a $0.1-\mathrm{cm}$-gap cuvette. The transformants were recovered in $200 \mu \mathrm{L}$ SOC medium and plated on $\mathrm{LB}(0.5 \% \mathrm{NaCl})$ agar medium containing $50 \mathrm{\mu g} / \mathrm{mL}$ zeocin and $50 \mathrm{\mu g} / \mathrm{mL}$ kanamycin. The plated dishes were incubated at $37^{\circ} \mathrm{C}$ overnight, and 96 or 192 single colonies were isolated and cultured in Terrific broth (TB) medium (9) containing the antibiotics described above.

Each pDONR-Zeo plasmid DNA extracted from the pool was subjected to sequencing reactions in 384-well plates with KAN-2 transposon-specific sequencing primers (FP-1, 5'-ACCTACAACAAAGCTCTCATCAACC-3'; RP-4, 5'-TGTA AGCAGACAGTTTTATTG- $\left.3^{\prime}\right)$. Sequencing reactions were also performed for each unpooled cDNA clone with primers specific to the pCMVFL 3 vector sequence [designated as universal primers: forward, 


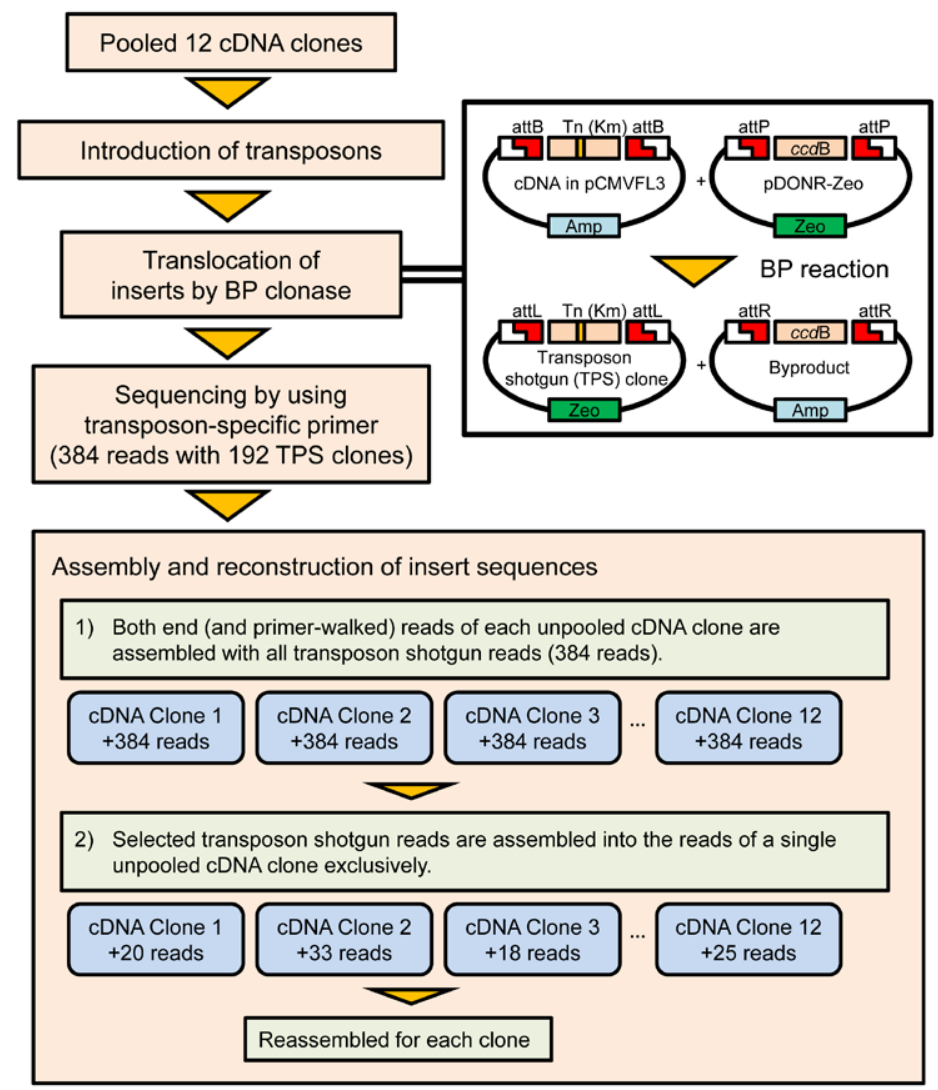

Figure 1. Chart of the computational analysis procedure used to reconstruct cDNA sequences from the results of TPS sequencing. In the example shown, 12 cDNA clones were pooled, and 192 TPS clones were produced per cDNA clone pool. Numbers of TPS reads in the second assembly step are presented as an example.

5'-ATTTAGGTGACACTATAGAA-3'; reverse, 5'-TAATACGACTCACTATAGGG-3'; reverse poly $\left.(\mathrm{dT}), \mathrm{T}_{25} \mathrm{~V}\right]$. Sequencing was performed with BigDye terminator v3.1, an ABI9700 thermal cycler, and an ABI3730XL sequencer (all from Applied Biosystems, Foster City, CA, USA).

The sequencing chromatograms were base called by Phred, and the DNA sequences were assembled into contigs by Phrap $(10,11)$, with default parameters and masking of vector and transposon sequences. Those TPS sequences that did not form part of the same contig as sequences obtained from the unpooled cDNA clones by the universal primers

were discarded, and the final assembly was conducted in their absence. A schema of the computational analysis is presented in Figure 1. When all bases possessed Phred quality values $>25$, and there were no ambiguous bases after manual inspection using Consed (12), we considered the cDNA clones to be completely sequenced. When the reconstructed cDNA clones were aligned with the draft sequence of the pig genome (13), we observed no unnatural organization of exons (data not shown).

We evaluated the TPS sequencing efficiency with the BP reaction. When the transposon-specific primer (FP-1 or RP-4) generated a sequencing read

Table 2. Success rates of TPS sequencing with or without the BP reaction for pooled cDNA clones.

\begin{tabular}{|lccc|}
\hline & Without BP reaction & With BP reaction \\
\hline $\begin{array}{l}\text { Number of TPS clones } \\
\text { Mean number of specifically aligned TPS reads per } \\
\text { CDNA clone }( \pm \text { SD })\end{array}$ & $\mathbf{9 6}$ & $\mathbf{1 9 2}$ & $\mathbf{9 6}$ \\
\hline $\begin{array}{l}\text { Number of completed cDNA sequences } \\
5.80 \pm 0.58\end{array}$ & $12.08 \pm 0.11$ & $13.07 \pm 0.08$ \\
\hline
\end{tabular}

Twenty pools, each consisting of 12 cDNA clones ( 240 clones in total), were subjected to transposon shotgun (TPS) sequencing. CDNA sequences were reconstructed by the assembly of specifically aligned TPS reads and sequencing reads of each of the unpooled cDNA clones.

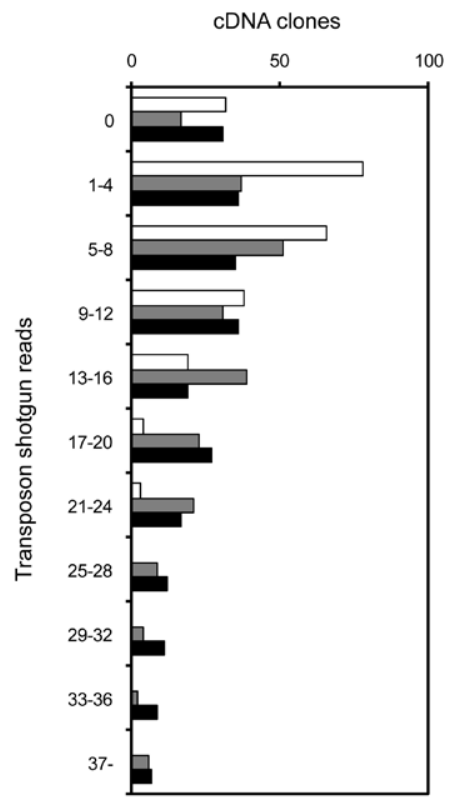

Figure 2. Distribution of numbers of TPS sequencing reads specifically aligned to cDNA clones. Twenty cDNA pools, each containing 12 cDNA clones (240 cDNA clones in total) were used. Numbers of cDNA clones possessing specifically aligned TPS sequencing reads when 96 TPS clones were generated with (black bars) or without (white bars) the BP reaction and subjected to bidirectional shotgun sequencing are indicated (192 reads per cDNA clone). Results for 192 TPS clones without the BP reaction are also indicated (gray bars)

without the vector sequence, the corresponding TPS clone was classed as "within insert." When the sequencing read contained the vector sequence, the corresponding TPS clone was classed as "within vector." Without the BP reaction, the proportion of clones "within insert" was $35 \%$ (Table 1), which is more than the proportion estimated from the average insert size $(2.0 \mathrm{~kb})$ shown by electrophoresis $(29 \%)$ (data not shown), probably because collapse of the replication initiation site (ori) or antibiotic resistance gene(s) results in failure of replication of the plasmid (5). Approximately two-thirds of the TPS clones before the $\mathrm{BP}$ reaction were not informative because the transposon insertion was within the vector sequences. However, more than $90 \%$ of the clones were confirmed as "within insert" after the BP reaction (Table 1).

We reconstructed the cDNA sequences of 20 pools, each consisting of 12 cDNA clones, by assembling TPS sequencing reads of clones that had been subjected to, or had not been subjected to, the BP reaction. When 96 TPS clones 
were generated for each clone pool, and sequencing reads from both directions (192 reads for each pool) were assembled with reads from the unpooled cDNA clones using universal primers, the number of reads specifically aligned with each cDNA clone from TPS sequencing with the $\mathrm{BP}$ reaction (TPS-BP) was more than double the number without the $\mathrm{BP}$ reaction. The number of cDNA clones with specifically aligned TPS sequencing reads when we used 96 TPS clones with the BP reaction was more than when we used 192 TPS clones without the BP reaction (Figure 2 and Table 2).

We considered that TPS-BP might be a useful strategy for clones that could not be completely sequenced by the primerwalking method alone. Therefore, we conducted TPS-BP for $180 \mathrm{cDNA}$ clones that had twice been subjected to primerwalking (the average number of custom sequencing primers per cDNA clone was 5.4) but had not been completely sequenced in our sequencing project (7). The cDNA clones were allocated to 15 pools, each consisting of 12 clones. TPS clones (192 clones per pool) of the mixed plasmid DNA of each pool were generated with the BP reaction and subjected to bidirectional sequencing. The combination of reads using the TPS-BP and primer-walking methods slightly increased the number of completely sequenced cDNA clones (Supplementary Table S1). Our results demonstrated that the combined method was preferable to either method alone, although TPS-BP alone was sufficient for $\mathrm{cDNAs}$ that were difficult to completely sequence.

The modification of TPS-BP described here has a benefit of reducing the number of shotgun clones to be sequenced, and it may be applicable to large-scale and highly automated cDNA sequencing projects with reduced costs.

\section{Acknowledgments}

We thank Toshie Iioka and Takako Suzuki for their technical assistance. This study was supported in part by the Selective Research Support Program of the National Institute of Agrobiological Sciences, the Animal Genome Research Project of the Ministry of Agriculture, Forestry, and Fisheries of Japan, and a Grant-in-Aid from the Japan Racing Association.

\section{Competing interests}

The authors declare no competing interests.

\section{References}

1. Wiemann, S., B. Weil, R. Wellenreuther, J. Gassenhuber, S. Glassl, W. Ansorge, M. Böcher, H. Blöcker, et al. 2001. Toward a catalog of human genes and proteins: sequencing and analysis of 500 novel complete protein coding human cDNAs. Genome Res. 11:422-435.

2.Devine, S.E., S.L. Chissoe, Y. Eby, R.K. Wilson, and J.D. Boeke. 1997. A transposon-based strategy for sequencing repetitive DNA in eukaryotic genomes. Genome Res. 7:551-563.

3. Butterfield, Y.S., M.A. Marra, J.K. Asano, S.Y. Chan, R. Guin, M.I. Krzywinski, S.S. Lee, K.W. MacDonald, et al. 2002. An efficient strategy for large-scale high-throughput transposon-mediated sequencing of cDNA clones. Nucleic Acids Res. 30:2460-2468.

4. Hartley, J.L., G.F. Temple, and M.A. Brasch. 2000. DNA cloning using in vitro site-specific recombination. Genome Res. 10:1788-1795.

5. Shevchenko, Y., G.G. Bouffard, Y.S. Butterfield, R.W. Blakesley, J.L. Hartley, A.C. Young, M.A. Marra, S.J. Jones, et al. 2002. Systematic sequencing of cDNA clones using the transposon Tn5. Nucleic Acids Res. 30:2469-2477.

6. Uenishi, H., T. Eguchi, K. Suzuki, T. Sawazaki, D. Toki, H. Shinkai, N. Okumura, N. Hamasima, and T. Awata. 2004. PEDE (Pig EST Data Explorer): construction of a database for ESTs derived from porcine fulllength cDNA libraries. Nucleic Acids Res. 32:D484-D488.
7. Uenishi, H., T. Eguchi-Ogawa, H. Shinkai, N. Okumura, K. Suzuki, D. Toki, N. Hamasima, and T. Awata. 2007. PEDE (Pig EST Data Explorer) has been expanded into Pig Expression Data Explorer, including 10,147 porcine full-length cDNA sequences. Nucleic Acids Res. 35:D650D653.

8. Suzuki, Y., K. Yoshitomo-Nakagawa, K. Maruyama, A. Suyama, and S. Sugano. 1997. Construction and characterization of a full length-enriched and a 5 -end-enriched cDNA library. Gene 200:149-156.

9. Sambrook, J. and D.W. Russell. 2001. Molecular Cloning, 3rd ed. CSH Laboratory Press, Cold Spring Harbor, NY.

10. Ewing, B. and P. Green. 1998. Base-calling of automated sequencer traces using phred. II. Error probabilities. Genome Res. 8:186-194.

11. Ewing, B., L. Hillier, M.C. Wendl, and P. Green. 1998. Basecalling of automated sequencer traces using phred. I. Accuracy assessment. Genome Res. 8:175-185

12. Gordon, D., C. Abajian, and P. Green. 1998. Consed: a graphical tool for sequence finishing. Genome Res. 8:195-202.

13. Archibald, A.L., L. Bolund, C. Churcher, M. Fredholm, M.A. Groenen, B. Harlizius, K.T. Lee, D. Milan, et al. 2010. Pig genome sequence-analysis and publication strategy. BMC Genomics 11:438.

Received 10 May 2011; accepted 14 July 2011.

Address correspondence to Hirohide Uenishi, Agrogenomics Research Center, National Institute of Agrobiological Sciences, 2 Ikenodai, Tsukuba, Ibaraki 305-8602, Japan.

To purchase reprints of this article, contact: biotechniques@fosterprinting.com

\section{www.eEnzyme.com}

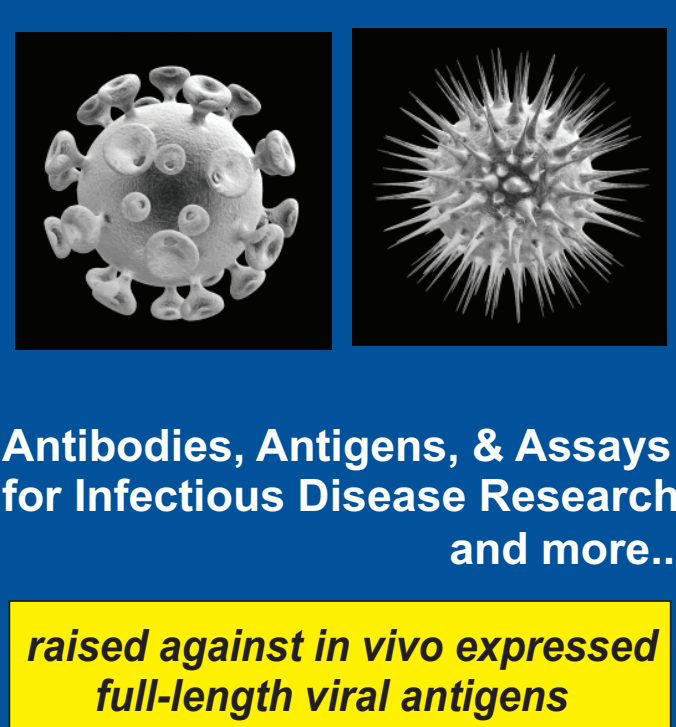

expressed in human cell lines

Gaithersburg, Maryland 1800.919 .0755 